\title{
Ginsenoside Rc Is a New Selective UGT1A9 Inhibitor in Human Liver Microsomes and Recombinant Human UGT Isoforms ${ }^{\text {[ }}$
}

\author{
Hyunyoung Lee, Jae-Kyung Heo, Ga-Hyun Lee, So-Young Park, Su-Nyeong Jang, Hyun-Ji Kim, \\ Mi Jeong Kwon, Im-Sook Song, and Kwang-Hyeon Liu
}

BK21 Plus KNU Multi-Omics based Creative Drug Research Team, College of Pharmacy and Research Institute of Pharmaceutical Sciences, Kyungpook National University, Daegu, South Korea

Received May 14, 2019; accepted September 25, 2019

\begin{abstract}
Ginseng is known to have inhibitory effects on UGT1A9 activity. However, little is known about the inhibitory effects of ginsenosides, the major active compounds in ginseng, on UGT1A9 activity. In vitro investigation of UGT1A9 inhibition by ginsenosides was carried out using human liver microsomes (HLMs). Among 10 ginsenosides, ginsenoside Rc was the strongest inhibitor of UGT1A9-mediated mycophenolic acid glucuronidase activity. Further inhibition kinetic studies using HLMs suggested that ginsenoside Rc competitively and noncompetitively inhibited UGT1A9-mediated propofol and mycophenolic acid glucuronidation activities, with $K_{\mathrm{i}}$ values of 2.83 and $3.31 \mu \mathrm{M}$, respectively. Next, to investigate whether the inhibitory effect of ginsenoside Rc is specific to the UGT1A9 isoform, we studied the inhibitory potency of ginsenoside Rc on nine human uridine diphospho-glucuronosyltransferase (UGT) activities using recombinant human UGT isoforms. Ginsenoside Rc exhibited a 12.9-fold selectivity (which was similar to niflumic acid at 12.5-fold) for UGT1A9 inhibition. Ginsenoside Rc at $50 \mu \mathrm{M}$
\end{abstract}

also inhibited none of the other UGT isoform-specific activities above $12.0 \%$, except for UGT1A9 (>91.5\%) in HLMs, indicating that ginsenoside Rc might be used as a selective UGT1A9 inhibitor in reaction phenotyping studies of new chemical entities. Considering lower plasma concentrations $(0.01 \mu \mathrm{M})$ of ginsenoside $R c$ in healthy subjects and no induction potential on UGT isoforms, ginsenoside $\mathrm{Rc}$ does not cause pharmacokinetic drug interactions with other coadministered drugs metabolized by UGT1A9.

\section{SIGNIFICANCE STATEMENT}

Ginsenoside Rc selectively inhibited UGT1A9-mediated propofol and mycophenolic acid glucuronidation activities in human liver microsomes and recombinant uridine diphospho-glucuronosyltransferase (UGT) isoforms. It exhibited a 12.9-fold selectivity for UGT1A9 inhibition. Therefore, ginsenoside Rc might be used as a selective UGT1A9 inhibitor in reaction phenotyping studies of new chemical entities, such as niflumic acid.

\section{Introduction}

Ginseng, one of the most popular medicinal herbs, has been widely used for thousands of years in Asia and has been increasingly consumed as a dietary supplement in Europe and the United States of America (Attele et al., 1999; Fang et al., 2013). A survey of herbal-based overthe-counter medicines showed that $28 \%$ of such preparations contain ginseng (Tyler, 2000). A variety of pharmacological effects have been ascribed to ginseng, including antiaging, anticancer, antidiabetic, antifatigue, antioxidant, cardioprotective, homeostatic, immunostimulatory, neuroprotective, and stress-reduction effects (Chen et al., 2008; Qi et al., 2011). Many studies have demonstrated that most of the pharmacological effects of ginseng are attributable to ginsenosides (Qi et al., 2011). Coadministration of ginseng with prescribed drugs is common. Therefore, significant herb-drug

This research was supported by the National Research Foundation of Korea, Ministry of Science and ICT, Republic of Korea [Grant NRF-2019R1A2C1008713], and by a Korea Basic Science Institute National Research Facilities \& Equipment Center grant funded by the Korea government (Ministry of Education) [Grant 2019R1A6C1010001].

https://doi.org/10.1124/dmd.119.087965.

SThis article has supplemental material available at dmd.aspetjournals.org. interactions between ginseng and therapeutic drugs, including ginsengwarfarin (Vaes and Chyka, 2000; Cheng, 2005) and ginseng-fexofenadine interactions (Kim et al., 2018), have been reported. These herb-drug interactions can affect the pharmacokinetics of drugs in humans and alter their pharmacological effects by regulating metabolizing enzymes or transporters.

To date, there have been several reports about the effects of ginseng on cytochrome P450, a representative phase I metabolizing enzyme. Kawase et al. (2009) reported that ginseng extracts significantly increased the expression levels of CYP1A1 and CYP3A11 in rat hepatocytes. In addition to animal studies, repeated administration of Panax ginseng capsules also resulted in the reduction of plasma midazolam concentration, indicating the possible induction of CYP3A in humans (Malati et al., 2012). Bilgi et al. (2010) reported that ginseng was associated with the occurrence of imatinib-induced liver toxicity after concurrent administration of ginseng in chronic myeloid leukemia patients, indicating the inhibition of CYP3A and P-glycoprotein, major imatinib-metabolizing enzyme and transporter, respectively. Smith et al.(Smith et al., 2001) also found ginseng to increase the plasma concentration of nifedipine, a CYP3A4 substrate drug, when coadministered. Uridine diphospho-glucuronosyltransferase (UGT) inhibition is also regarded as one of the most important factors for

ABBREVIATIONS: HLM, human liver microsome; IS, internal standard; LC-MS/MS, liquid chromatography-tandem mass spectrometry; 3-MC, 3methylcholanthrene; PPD, protopanaxadiol; PPT, protopanaxatriol; rUGT, recombinant UGT; SN-38, 7-ethyl-10-hydroxy-camptothecin; UDPGA, uridine 5'-diphosphoglucuronic acid ammonium salt; UGT, uridine diphospho-glucuronosyltransferase. 


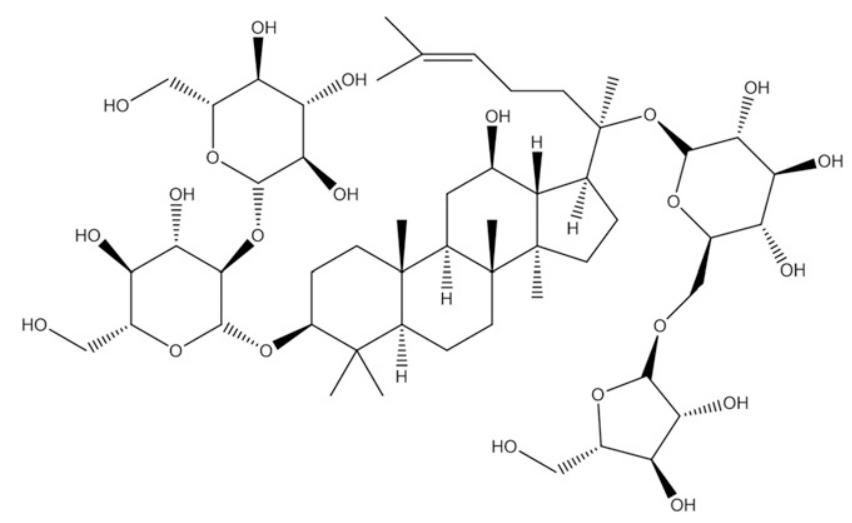

Fig. 1. Chemical structure of ginsenoside Rc.

clinical drug interactions (Sahai et al., 1994; Kiang et al., 2005; Zhang et al., 2005; Oda et al., 2015). The common modulatory effect on UGT activity is because glucuronidation accounts for more than $35 \%$ of phase II drug metabolism (Kiang et al., 2005; Mroz et al., 2018). Recently, two studies investigating UGT-based ginseng-drug interactions have been reported. Ginseng root extracts were demonstrated to have inhibitory effects on UGT1A9 and UGT1A4 (Mohamed and Frye, 2011), with $\mathrm{IC}_{50}$ values of 298.6 and $368.4 \mu \mathrm{g} / \mathrm{ml}$, respectively. Additionally, purified dry extracts of ginseng exhibited inhibition against human UGT1A1, UGT1A9, and UGT2B7 activities, with $\mathrm{IC}_{50}$ values ranging from 14.5 to $31.5 \mu \mathrm{g} / \mathrm{ml}$ (Zheng et al., 2014). These two studies indicated that ginseng had inhibitory effects on UGT1A9 activity. However, few data are available regarding the inhibitory effects of ginsenosides, major active compounds in ginseng, against UGT1A9 activity. Fang et al. (2013) reported that $100 \mu \mathrm{M}$ ginsenosides $\mathrm{Rb} 2, \mathrm{Rc}$, and $\mathrm{Rg} 3$ inhibited more than $60 \%$ of recombinant UGT1A9-mediated 4-methylumbelliferone glucuronidase activity. Ginsenoside Rg3 inhibited human liver microsomal UGT1A9 activity with an $\mathrm{IC}_{50}$ value of $15.1 \mu \mathrm{M}$, whereas ginsenosides $\mathrm{Rg} 2, \mathrm{Rh} 1$, and $\mathrm{Rh} 2$ had negligible inhibitory effects on UGT1A9 $\left(\mathrm{IC}_{50}>100 \mu \mathrm{M}\right)$ (Kim et al., 2016).

In this study, we evaluated the inhibitory effects of ginsenosides F1, F2, Rb1, Rb2, Rc, Rd, Re, Rf, Rg1, and Rg3 on UGT1A9 using human liver microsomes (HLMs) to investigate specifically which ginsenosides in ginseng are involved in UGT1A9 inhibition. We also investigated the inhibitory potency of ginsenoside Rc (Fig. 1) against the action of eight other UGT isoforms to determine the UGT1A9 selectivity of ginsenoside Rc, which most strongly inhibited UGT1A9 activity. The availability of a selective UGT1A9 inhibitor would be beneficial for assessing the extent of UGT1A9's contributions to phase II drug metabolism. Furthermore, the inhibition mechanism and kinetic parameters $\left(K_{\mathrm{i}}\right)$ were determined for ginsenoside Rc and compared with those of niflumic acid, a well known selective UGT1A9 inhibitor (Miners et al., 2011).

\section{Materials and Methods}

Chemicals and Reagents. Androsterone, carvedilol, chenodeoxycholic acid, hecogenin, estrone- $\beta$-D-glucuronide sodium salt, $N$-acetylserotonin, naloxone, niflumic acid, mefenamic acid, 3-methylcholanthrene (3-MC), testosterone, trifluoperazine, and uridine $5^{\prime}$-diphosphoglucuronic acid ammonium salt (UDPGA) were obtained from Sigma-Aldrich (St. Louis, MO). 7-Ethyl10-hydroxy-camptothecin (SN-38) was provided by Santa Cruz Biotechnology (Dallas, TX). Atazanavir, celastrol, mycophenolic acid, mycophenolic acid $\beta$-D-glucuronide, propofol, propofol $\beta$-D-glucuronide, and troglitazone were purchased from Toronto Research Chemicals (Toronto, ON, Canada). Ginsenoside F1 (98.2\%), ginsenoside F2 (98.4\%), ginsenoside Rb1 (99.0\%), ginsenoside $\mathrm{Rb} 2$ (100\%), ginsenoside Rc (100\%), ginsenoside Rd (98.9\%), ginsenoside $\operatorname{Re}(100 \%)$, ginsenoside Rf (95.0\%), ginsenoside Rg1 (99.7\%), and ginsenoside $\operatorname{Rg} 3$ (98.5\%) were obtained from Ambo Institute (Daejeon, Korea). All solvents were liquid chromatography-mass spectrometry grade (Fisher Scientific Co., Pittsburgh, PA). All the other reagents were of analytical or liquid chromatography-mass spectrometry grade and are commercially available. The pooled HLMs (XTreme 200), which were made up of equal numbers of male and female livers, were obtained from XenoTech (Lenexa, KS). And recombinant human UGT isoforms (UGT1A1, UGT1A3, UGT1A4, UGT1A6, UGT1A9, UGT2B4, UGT2B7, UGT2B15, and UGT2B17) were purchased from BD Biosciences (Woburn, MA) and were stored at $-80^{\circ} \mathrm{C}$ until use. Cryopreserved human hepatocytes (HFC520, lot number 321, female, age 58, Caucasian, smoker) were purchased from Corning Life Sciences (Woburn, MA) and were stored in liquid nitrogen until use.

Inhibitory Effects of 10 Ginsenosides against UGT1A9 Activity. Ten ginsenosides $(0-50 \mu \mathrm{M})$ were screened for inhibition of human liver microsomal UGT1A9 activity. Each ginsenoside stock solution was prepared in methanol such that the final concentration of solvent in the incubation mixtures was $1 \%(\mathrm{v} / \mathrm{v})$, which has a negligible or minor effect on most UGT activities (Uchaipichat et al., 2004). The microsomal incubations were performed in a $100-\mu \mathrm{l}$ reaction mixture containing $0.25 \mathrm{mg} / \mathrm{ml}$ microsomal protein, $25 \mu \mathrm{g} / \mathrm{ml}$ alamethicin, $10 \mathrm{mM} \mathrm{MgCl}, 0.1 \mathrm{M}$ tris buffer ( $\mathrm{pH} 7.4$ ), and $0.2 \mu \mathrm{M}$ mycophenolic acid, which is UGT1 A9-specific substrate (Joo et al., 2014). UDPGA ( $5 \mathrm{mM})$ was added after preincubation $\left(37^{\circ} \mathrm{C}, 5\right.$ minutes). Following incubation at $37^{\circ} \mathrm{C}$ for 1 hour in a thermo-shaker, the reactions were terminated by adding $50 \mu \mathrm{l}$ of cold acetonitrile containing $0.25 \mu \mathrm{M}$ estrone glucuronide [internal standard (IS)] into the reaction mixture. After centrifugation $\left(10,000 \mathrm{~g}, 4^{\circ} \mathrm{C}, 5\right.$ minutes), aliquots of the supernatant were analyzed by liquid chromatography-tandem mass spectrometry (LC-MS/MS; Shimadzu LCMS 8060 system; Shimadzu, Kyoto, Japan) as described previously (Joo et al., 2014).

Ginsenoside Rc Inhibition of Human Liver Microsomal UGT Activity. All microsomal incubations were conducted in triplicate under linear incubation time for the formation of metabolites. Ginsenoside $\operatorname{Rc}(0,0.5,2,5,10,20,50$, and $100 \mu \mathrm{M})$ was screened for inhibition of human liver microsomal UGT1A1, 1A3, $1 \mathrm{~A} 4,1 \mathrm{~A} 6,1 \mathrm{~A} 9$, and $2 \mathrm{~B} 7$ activities, and the UGT inhibitory potential of ginsenoside Rc was compared with that of niflumic acid $(0,0.2,0.5,1,2,5$, and $10 \mu \mathrm{M}$ ), a known selective UGT1A9 inhibitor (Miners et al., 2011). A previously developed substrate cocktail method that enables the simultaneous

TABLE 1

Substrates, their metabolites, and their LC-MS/MS conditions for human UGT assays

\begin{tabular}{|c|c|c|c|c|c|c|}
\hline UGT enzyme & Substrate & Conc. $(\mu \mathrm{M})$ & Metabolites & Transition $(\mathrm{m} / \mathrm{z})$ & Mode & $\mathrm{CE}(\mathrm{eV})$ \\
\hline $1 \mathrm{~A} 1$ & SN-38 & 0.5 & SN-38 glucuronide & $569>393$ & + & 30 \\
\hline $1 \mathrm{~A} 3$ & Chenodeoxycholic acid & 2.0 & Chenodeoxycholic acid 24-acyl glucuronide & $567>391$ & - & 20 \\
\hline $1 \mathrm{~A} 4$ & Trifluoperazine & 0.5 & Trifluoperazine $N$-glucuronide & $584>408$ & + & 30 \\
\hline 1A6 & $\mathrm{N}$-Acetylserotonin & 1 & $\mathrm{~N}$-Acetylserotonin glucuronide & $395>219$ & + & 10 \\
\hline 1A9 & Mycophenolic acid & 0.2 & Mycophenolic acid glucuronide & $495>319$ & - & 25 \\
\hline 2B4 & Carvedilol & 1.0 & Carvedilol glucuronide & $583>407$ & + & 25 \\
\hline 2B7 & Naloxone & 1.0 & Naloxone 3-glucuronide & $504>310$ & + & 30 \\
\hline 2B15 & Testosterone & 50 & Testosterone 17 -glucuronide & $465>97$ & + & 27 \\
\hline 2B17 & Androsterone & 0.5 & Androsterone 3-glucuronide & $465>289$ & - & 35 \\
\hline
\end{tabular}

$\mathrm{CE}$, collision energy; Conc., concentration. 
TABLE 2

Inhibitory potential of 10 ginsenosides against UGT1A9-mediated mycophenolic acid glucuronidase activity in human liver microsomes

\begin{tabular}{|c|c|c|c|c|c|c|c|c|c|c|}
\hline \multirow{2}{*}{ Inhibitor } & \multicolumn{6}{|c|}{ Protopanaxadiol Type } & \multicolumn{4}{|c|}{ Protopanaxatriol Type } \\
\hline & $\mathrm{F} 2$ & $\mathrm{Rb} 1$ & $\mathrm{Rb} 2$ & $\mathrm{Rc}$ & $\mathrm{Rd}$ & $\operatorname{Rg} 3$ & F1 & $\operatorname{Re}$ & $\mathrm{Rf}$ & $\operatorname{Rg} 1$ \\
\hline $\mathrm{IC}_{50}(\mu \mathrm{M})^{a}$ & 44.0 & 21.3 & 22.7 & 6.3 & 17.4 & 10.2 & $>50$ & $>50$ & $>50$ & $>50$ \\
\hline
\end{tabular}

${ }^{a}$ Values represent the average of triplicate.

incubation and measurement of compound inhibitory potential against each UGT isoform was used to obtain $\mathrm{IC}_{50}$ values (Joo et al., 2014). The selective UGT isoform substrates were used at concentrations approximately equal to their respective Michaelis-Menten constant $\left(K_{\mathrm{m}}\right)$ values: $0.5 \mu \mathrm{M}$ for SN-38 (UGT1A1), $2 \mu \mathrm{M}$ for chenodeoxycholic acid (UGT1A3), $0.5 \mu \mathrm{M}$ for trifluoperazine (UGT1A4), $1 \mu \mathrm{M}$ for $N$-acetylserotonin (UGT1A6), $0.2 \mu \mathrm{M}$ for mycophenolic acid (UGT1A9), and $1 \mu \mathrm{M}$ for naloxone (UGT2B7) (Joo et al., 2014). Known inhibitors also were included as positive controls to validate the experiments. Atazanavir (0-20 $\mu \mathrm{M})$, celastrol (0-50 $\mu \mathrm{M})$, hecogenin $(0-50 \mu \mathrm{M})$, troglitazone $(0-20 \mu \mathrm{M})$, niflumic acid $(0-10 \mu \mathrm{M})$, and mefenamic acid $(0-50 \mu \mathrm{M})$ were used as inhibitors of UGTs 1A1, 1A3, 1A4, 1A6, 1A9, and 2B7, respectively (Joo et al., 2014). The microsomal incubations were performed in a $100-\mu 1$ reaction mixture containing $0.25 \mathrm{mg} / \mathrm{ml}$ microsomal protein (the protein concentration was in the linear range of metabolite formation), $25 \mu \mathrm{g} / \mathrm{ml}$ alamethicin, $10 \mathrm{mM} \mathrm{MgCl} 2,0.1 \mathrm{M}$ tris buffer ( $\mathrm{pH} 7.4$ ), and each UGT isoform-specific individual substrate. UDPGA (5 mM) was added after preincubation $\left(37^{\circ} \mathrm{C}, 5\right.$ minutes $)$. Following incubation at $37^{\circ} \mathrm{C}$ for 1 hour in a thermo-shaker, the reactions were terminated by adding $50 \mu \mathrm{l}$ of cold acetonitrile containing $0.25 \mu \mathrm{M}$ estrone glucuronide (IS) into the reaction mixture. After centrifugation $\left(10,000 \mathrm{~g}, 4^{\circ} \mathrm{C}, 5\right.$ minutes $)$, aliquots of the supernatant were analyzed by LC-MS/MS (Shimadzu LCMS 8060 system; Shimadzu) as described previously (Joo et al., 2014). The selected reaction monitoring transitions and collision energies determined for each metabolite are listed in Table 1.

Ginsenoside Rc Inhibition of Recombinant UGT Enzyme Activity. Ginsenoside Rc was screened for inhibition of recombinant UGT1A1, 1A3, 1A4, 1A6, 1A9, 2B4, 2B7, 2B15, and 2B17 activities using the selective substrates as probes. The selective UGT isoform substrates were used at concentrations approximately equal to their respective Michaelis-Menten constant $\left(K_{\mathrm{m}}\right)$ values: $0.5 \mu \mathrm{M}$ for SN-38 (UGT1A1), $2 \mu \mathrm{M}$ for chenodeoxycholic acid (UGT1A3), $0.5 \mu \mathrm{M}$ for trifluoperazine (UGT1A4), $1 \mu \mathrm{M}$ for $N$-acetylserotonin (UGT1A6), $0.2 \mu \mathrm{M}$ for mycophenolic acid (UGT1A9), $1 \mu \mathrm{M}$ for carvedilol (UGT2B4) (Ohno et al., 2004), $1 \mu \mathrm{M}$ for naloxone (UGT2B7), $50 \mu \mathrm{M}$ for testosterone (UGT2B15) (Sten et al., 2009a), and $0.5 \mu \mathrm{M}$ for androsterone (UGT2B17) (Sten et al., 2009b). Incubation mixtures (100 $\mu \mathrm{l})$ contained recombinant UGT (rUGT; final concentration: $0.25 \mathrm{mg} / \mathrm{ml}$ ), $5 \mathrm{mM}$ UDPGA, $10 \mathrm{mM} \mathrm{MgCl} 2,100 \mathrm{mM}$ Tris- $\mathrm{HCl}$ buffer ( $\mathrm{pH}$ 7.4), and UGT isoform probe substrate in the absence or presence of ginsenoside Rc $(0,0,5,2,5,10,20$, and $50 \mu \mathrm{M})$. After a 5-minute preincubation period, UDPGA was added to the mixture to initiate the reaction. Following incubation at $37^{\circ} \mathrm{C}$ for 1 hour, the reactions were terminated by adding cold acetonitrile containing IS. After centrifugation, aliquots of the supernatant were analyzed by LC-MS/MS as described previously (You et al., 2007; Hauser et al., 2008; Sten et al., 2009b; Joo et al., 2014). The selected reaction monitoring transitions and collision energies determined for each metabolite are listed in Table 1.

Kinetic Characterization of Ginsenoside Rc Inhibition of UGT1A9 in Human Liver Microsomes. Mechanisms of inhibition and inhibition constants ( $K_{\mathrm{i}}$ values) for ginsenoside Rc inhibition of UGT1A9 were determined with pooled HLMs and recombinant human UGT1A9 enzyme (rUGT1A9). Incubation conditions were as described in the previous section. Propofol and mycophenolic acid were used as the UGT1A9-selective probe substrates. To determine the inhibition constant of ginsenoside Rc in HLMs, different concentrations of ginsenoside $\operatorname{Rc}(0,2,5,10,20,50$, and $100 \mu \mathrm{M})$ were added to reaction mixtures containing different concentrations of propofol (10, 20, and $50 \mu \mathrm{M})$ or mycophenolic acid $(0.1,0.2$, and $0.5 \mu \mathrm{M})$. After a 1-hour incubation period, the reactions were terminated by the addition of cold acetonitrile containing IS. After centrifugation, aliquots of the supernatant were analyzed by LC-MS/MS as described previously (Joo et al., 2014; Seo et al., 2014). The $K_{\mathrm{i}}$ values of ginsenoside Rc were compared with those of niflumic acid $(0,0.2,0.5,1,2,5$, and $10 \mu \mathrm{M})$, a known UGT1A9 inhibitor.

Effect of Ginsenoside Rc on the mRNA Levels of UGT Isoforms. Effects of ginsenoside Rc on mRNA levels of UGT1A1, UGT1A4, UGT1A9, and UGT2B7 were assessed in cryopreserved human hepatocyte cultures as described previously (Seong et al., 2018). In brief, cryopreserved human hepatocytes were thawed, and viable cells $\left(4 \times 10^{5}\right)$ were seeded in 24-well plates precoated with collagen type I and cultured with matrigel overlay. The cells were treated with vehicle $(0.1 \%$ DMSO), 3-MC $(5 \mu \mathrm{M}$; positive control), and ginsenoside $\operatorname{Rc}(0.5,2.5,25$, and $50 \mu \mathrm{M})$ for 48 hours. After 48 hours, total RNA from hepatocytes was immediately isolated, and the RNA concentration and purity were determined by an absorbance test at OD260/280 nm. Reverse-transcription polymerase chain reaction was conducted using a TaqMan RNA-to- $\mathrm{C}_{\mathrm{T}}$ 1-Step Kit, TaqMan Gene Expression Assay Kit, and respective primers for UGT1A1, 1A4, 1A9, and 2B7 (Applied Biosystems, Foster city, CA). The relative gene expression rate was calculated using the $\Delta \mathrm{Ct}$ value compared with the vehicle control. Glyceraldehyde-3-phosphate dehydrogenase was used as an endogenous internal standard.

Determination of Rc in Human Hepatocytes Using LC-MS/MS. Cryopreserved human hepatocytes were treated with ginsenoside Rc $(0.5,5$, and $50 \mu \mathrm{M}$ ) for 48 hours with a procedure identical to that described earlier. After 48 hours, culture medium and hepatocytes were collected for the quantitation of ginsenoside Rc. Methanol $(300 \mu \mathrm{l})$ containing $0.05 \mathrm{ng} / \mathrm{ml}$ berberine (IS) was added to $50 \mu \mathrm{l}$ of samples, and the mixtures were vortexed for 15 minutes. After centrifugation $\left(10,000 \mathrm{~g}, 4^{\circ} \mathrm{C}, 5\right.$ minutes $)$, aliquots of the supernatant were analyzed using an Agilent 6470 Triple Quad LC-MS/MS system (Agilent, Wilmington, DE) as described previously (Jin et al., 2019).

Data Analysis. Analytical data were processed using Shimadzu LabSolution LCMS software. The $\mathrm{IC}_{50}$ values were calculated using WinNonlin software (Pharsight, Mountain View, CA). The apparent kinetic parameters for inhibitory activity $\left(K_{\mathrm{i}}\right)$ were estimated by graphical methods, such as Dixon and Lineweaver-Burk plots, and were more accurately determined by nonlinear least-squares regression analysis, on the basis of the best enzyme inhibition model using the WinNonlin software (Miners et al., 2011; Phuc et al., 2017). The models

TABLE 3

$\mathrm{IC}_{50}$ values of well known UGT inhibitors, ginsenoside Rc, and niflumic acid against the activities of six UGT isoforms in pooled human liver microsomes

\begin{tabular}{|c|c|c|c|c|c|}
\hline \multirow{2}{*}{ UGTs } & \multirow{2}{*}{ Substrate } & \multicolumn{4}{|c|}{$\mathrm{IC}_{50}{ }^{a}$} \\
\hline & & \multicolumn{2}{|c|}{ Well Known Inhibitors } & Ginsenoside Rc & Niflumic Acid \\
\hline & & & $\mu M$ & $\mu M$ & $\mu M$ \\
\hline UGT1A1 & SN-38 & Atazanavir & $1.22 \pm 0.51$ & $>200$ & $15.3 \pm 2.7$ \\
\hline UGT1A3 & Chenodeoxycholic acid & Celastrol & $5.88 \pm 2.31$ & $>200$ & $>50$ \\
\hline UGT1A4 & Trifluoperazine & Hecogenin & $0.97 \pm 0.45$ & $>200$ & $>50$ \\
\hline UGT1A6 & $N$-Acetylserotonin & Troglitazone & $11.8 \pm 4.7$ & $>200$ & $>50$ \\
\hline UGT1A9 & Mycophenolic acid & Niflumic acid & $0.95 \pm 0.10$ & $6.34 \pm 1.02$ & $0.95 \pm 0.10$ \\
\hline UGT2B7 & Naloxone & Mefenamic acid & $20.3 \pm 2.0$ & $>200$ & $>50$ \\
\hline
\end{tabular}

${ }^{a}$ Values represent the average \pm S.E. of triplicate. 


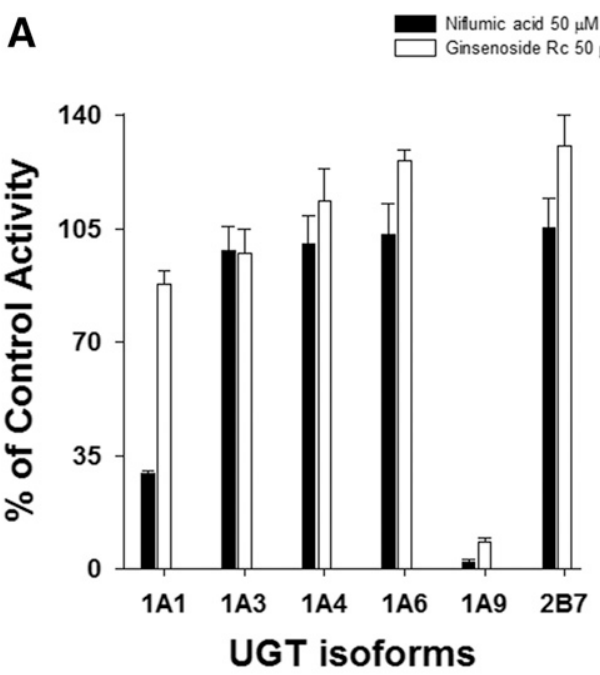

B

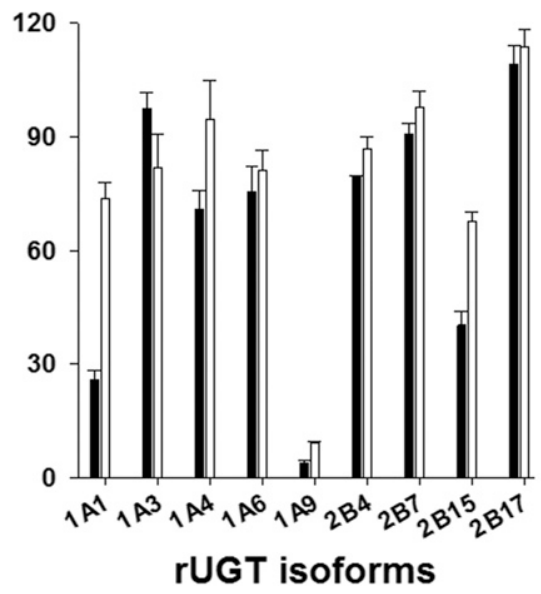

Fig. 2. Inhibitory effects of niflumic acid $(50 \mu \mathrm{M}, \mathbf{\square})$ and ginsenoside $\mathrm{Rc}(50 \mu \mathrm{M}, \square)$ on the enzymatic activities of six UGT isoforms in pooled human liver microsomes $(0.25 \mathrm{mg} / \mathrm{ml}$, H0630; XenoTech) (A) and nine UGT isoforms in recombinant UGTs $(0.25 \mathrm{mg} / \mathrm{ml}$; BD Biotechnology) (B). SN-38 $(0.5 \mu \mathrm{M})$, chenodeoxycholic acid $(2.0 \mu \mathrm{M})$, trifluoperazine $(0.5 \mu \mathrm{M})$, $N$-acetylserotonin $(1.0 \mu \mathrm{M})$, mycophenolic acid $(0.2 \mu \mathrm{M})$, carvedilol $(1.0 \mu \mathrm{M})$, naloxone $(1.0 \mu \mathrm{M})$, testosterone $(50 \mu \mathrm{M})$, and androsterone $(0.5 \mu \mathrm{M})$ were used as the probe substrates of UGTs $1 \mathrm{~A} 1$, $1 \mathrm{~A} 3,1 \mathrm{~A} 4,1 \mathrm{~A} 6,1 \mathrm{~A} 9,2 \mathrm{~B} 4,2 \mathrm{~B} 7,2 \mathrm{~B} 15$, and $2 \mathrm{~B} 17$, respectively. The data are shown as means of triplicate measurements $(n=3)$. tested included competitive, noncompetitive, uncompetitive, and mixed-type inhibition.

\section{Results}

Inhibition of UGT1A9 Activity by 10 Ginsenosides. The inhibitory potential of 10 ginsenosides against UGT1A9-mediated mycophenolic acid glucuronidation activity was evaluated using HLMs. Ginsenosides Rc, Rg3, Rd, Rb1, Rb2, and F2 inhibited UGT1 A9 activity with $\mathrm{IC}_{50}$ values of $6.3,10.2,17.4,21.3,22.7$, and $44.0 \mu \mathrm{M}$, respectively, whereas ginsenosides $\mathrm{F} 1$, Re, Rf, and $\mathrm{Rg} 1$ had no inhibitory effect on UGT1A9 ( $\mathrm{IC}_{50}>50 \mu \mathrm{M}$; Table 2$)$.

Inhibition of UGT Enzyme Activities by Ginsenoside Rc. The selectivity of ginsenoside Rc as an inhibitor of human UGTs was evaluated with HLMs. The $\mathrm{IC}_{50}$ values of known inhibitors of each UGT isoform were similar to previously reported values (Ito et al., 2001; Zhang et al., 2005; Uchaipichat et al., 2006; Choi et al., 2014; Joo et al., 2014; Zheng et al., 2014; Mendonza et al., 2016). Of the six UGT isoforms tested, ginsenoside Rc selectively inhibited UGT1A9catalyzed mycophenolic acid glucuronidation with an $\mathrm{IC}_{50}$ value of $6.34 \mu \mathrm{M}$, whereas it had no inhibitory effect on UGT1A1, 1A3, 1A4, 1A6, and 2B7 $\left(\mathrm{IC}_{50}>200 \mu \mathrm{M}\right.$; Table 3; Supplemental Fig. 1). Ginsenoside Rc at $50 \mu \mathrm{M}$ concentration inhibited none of the other UGT isoform-specific activities above $12.0 \%$, except for UGT1A9 (>91.5\%; Fig. 2A). Ginsenoside Rc showed slight activation for UGT1A6 and 2B7 isoforms; however, it did not show any inhibitory effects on these UGT isoforms at the concentration range tested $(0-50 \mu \mathrm{M})$. Niflumic acid, a known strong UGT1A9 inhibitor, inhibited UGT1A9-mediated mycophenolic acid glucuronidation and UGT1A1mediated SN-38 glucuronidation, with $\mathrm{IC}_{50}$ values of 0.95 and $15.3 \mu \mathrm{M}$, respectively (Table 3; Supplemental Fig. 1). The inhibition of UGT isoform activities by ginsenoside Rc was also examined up to $200 \mu \mathrm{M}$ using nine rUGT isoforms. Ginsenoside Rc at $50 \mu \mathrm{M}$ inhibited none of the other rUGT isoform-specific activities above 33\%, except for rUGT1A9 (>90\%; Fig. 2B). Ginsenoside Rc most strongly inhibited rUGT1A9-mediated mycophenolic acid glucuronidase activity, with an $\mathrm{IC}_{50}$ value of $6.38 \mu \mathrm{M}$, whereas it had weak inhibitory effects on rUGT2B7-mediated naloxone-glucuronidase activity $(82.2 \mu \mathrm{M})$ and rUGT1A1-mediated SN38-glucuronidase activity (98.9 $\mu \mathrm{M}$; Table 4). The $\mathrm{IC}_{50}$ values for ginsenoside $\mathrm{Rc}$ inhibition of UGTs 1A3, 1A4, 1A6, 2B4, 2B15, and 2B17 exceeded $100 \mu \mathrm{M}$ (Table 4). Niflumic acid at $50 \mu \mathrm{M}$ inhibited rUGT1A1 and rUGT2B15 to some extent $(>60 \%)$ and rUGT1A9 $(>95 \%$; Fig. 2$)$ to a greater extent.

Kinetic Characterization of Ginsenoside Rc Inhibition against UGT1A9 in Human Liver Microsomes. We further studied ginsenoside Rc to clarify its inhibition mechanism. Ginsenoside Rc inhibited UGT1A9-mediated mycophenolic acid glucuronidation activity, with a $K_{\mathrm{i}}$ value of $3.31 \pm 0.27 \mu \mathrm{M}$ (Table 5). The Dixon plots intersected the $x$-axis, indicating that ginsenoside Rc noncompetitively inhibited UGT1A9-mediated mycophenolic acid glucuronidase activity (Fig. 3A; Supplemental Fig. 2). To determine whether inhibition by ginsenoside Rc was substrate-specific, we also evaluated its inhibitory effects on UGT1A9-mediated propofol glucuronidation activity and found that ginsenoside Rc also markedly inhibited this activity, with a $K_{\mathrm{i}}$ value of $2.83 \pm 0.47 \mu \mathrm{M}$, in a competitive manner (Fig. 3B; Table 5). Niflumic acid inhibited UGT1A9-catalyzed mycophenolic acid glucuronidation and propofol glucuronidation activities, with $K_{\mathrm{i}}$ values of 0.72 and $1.07 \mu \mathrm{M}$, respectively, in a noncompetitive manner (Fig. 3B; Table 5).

Effect of Ginsenoside Rc on the mRNA Levels of UGT Isoforms. 3-MC, a positive control for UGT induction (Soars et al., 2004), increased the mRNA levels of UGT1A1, 1A4, 1A9, and 2B7 in human

TABLE 4

Inhibitory potential of niflumic acid and ginsenoside Rc against the activities of nine UGT isoforms in human recombinant UGT isoforms

Substrates were as follows: SN-38 (UGT1A1), chenodeoxycholic acid (UGTIA3), trifluoperazine (UGT1A4), N-acetylserotonin (UGT1A6), mycophenolic acid (UGT1A9), carvedilol (UGT2B4), naloxone (UGT2B7), testosterone (UGT2B15), and androsterone (UGT2B17)).

\begin{tabular}{|c|c|c|c|c|c|c|c|c|c|}
\hline \multirow{2}{*}{ Inhibitor } & \multicolumn{9}{|c|}{$\mathrm{IC}_{50}{ }^{a}$} \\
\hline & rUGT1A1 & rUGT1A3 & rUGT1A4 & rUGT1A6 & rUGT1A9 & rUGT2B4 & rUGT2B7 & rUGT2B15 & rUGT2B17 \\
\hline & $\mu M$ & $\mu M$ & $\mu M$ & $\mu M$ & $\mu M$ & $\mu M$ & $\mu M$ & $\mu M$ & $\mu M$ \\
\hline Niflumic acid & $17.5 \pm 1.1$ & $>50$ & $>50$ & $>50$ & $1.40 \pm 0.34$ & $>50$ & $>50$ & $29.2 \pm 4.8$ & $>50$ \\
\hline Ginsenoside Rc & $98.9 \pm 11.9$ & $111.5 \pm 12.3$ & $>200$ & $>200$ & $6.38 \pm 0.77$ & $156.5 \pm 3.5$ & $82.2 \pm 38.2$ & $104.0 \pm 15.1$ & $>200$ \\
\hline
\end{tabular}

${ }^{a}$ Values represent the average \pm S.E. of triplicate. 
TABLE 5

Apparent enzyme inhibition constants on the UGT1A9 activity by ginsenoside Rc and niflumic acid in human liver microsomes

The mean reaction rates of triplicate measurements were fitted into inhibition kinetic models.

\begin{tabular}{clc}
\hline Inhibitor & Mechanism of Inhibition & $K_{\mathrm{i}}{ }^{a}$ \\
\hline & & $\mu M$ \\
Mycophenolic acid & & \\
Ginsenoside Rc & Noncompetitive & $3.31 \pm 0.27$ \\
Niflumic acid & Noncompetitive & $0.72 \pm 0.06$ \\
Propofol & Competitive & $2.83 \pm 0.47$ \\
Ginsenoside Rc & Noncompetitive & $1.07 \pm 0.10$ \\
Niflumic acid & &
\end{tabular}

${ }^{a}$ Values are present as the average \pm S.E. of triplicate.

hepatocytes by 4.77-, 5.18-, 7.84-, and 5.86-fold, respectively, suggesting the feasibility of this induction system. However, ginsenoside Rc treatment $(0.5-50 \mu \mathrm{M})$ did not significantly alter the mRNA expression of four isoforms of UGT (Fig. 4). When the ginsenoside Rc concentration in the culture medium and hepatocytes were measured, ginsenoside Rc concentration in the culture medium was not significantly altered following 48-hour incubation, and the hepatocyte-to-medium ratio of ginsenoside Rc was 0.96-1.51, suggesting ginsenoside Rc would not accumulated in liver (Table 6).

\section{Discussion}

In the present study, the inhibitory effects of 10 ginsenosides (F1, F2, $\mathrm{Rb} 1, \mathrm{Rb} 2, \mathrm{Rc}, \mathrm{Rd}, \mathrm{Re}, \mathrm{Rf}, \mathrm{Rg} 1$, and $\mathrm{Rg} 3$ ) on human liver microsomal UGT1A9 activity were evaluated. Among 10 ginsenosides, ginsenoside Rc, a protopanaxadiol (PPD)-type ginsenoside, most strongly inhibited $\left(\mathrm{IC}_{50}=6.3 \mu \mathrm{M}\right)$ UGT1A9-mediated mycophenolic acid glucuronidase activity. Other PPD-type ginsenosides weakly inhibited UGT1A9 activity, with $\mathrm{IC}_{50}$ values ranging from 10.2 to $44.0 \mu \mathrm{M}$, whereas protopanaxatriol (PPT)-type ginsenosides (F1, Re, Rf, and Rg1) had negligible inhibitory effects on UGT1A9 $\left(\mathrm{IC}_{50}>50 \mu \mathrm{M}\right)$. These results were in concordance with earlier work, which had reported that PPDtype ginsenosides (Rb2, Rc, and $\mathrm{Rg} 3)$ at $100 \mu \mathrm{M}$ inhibited more than $60 \%$ of recombinant UGT1A9-mediated 4-methylumbelliferone glucuronidase activity, whereas PPT-type ginsenosides (F1, Re, Rg1, and Rh1) had no inhibitory effect on UGT1A9 (Fang et al., 2013). Kim et al. (2016) also reported that PPT-type ginsenosides, such as PPT, Rg2, and Rh1, had no inhibitory effects on human liver microsomal UGT1A9 activity $\left(\mathrm{IC}_{50}>100 \mu \mathrm{M}\right)$.

Next, we evaluated the inhibitory effects of ginsenoside Rc on nine human UGT isoforms by studying the activities of the UGT isoforms' marker reactions to determine the UGT1A9 selectivity of ginsenoside Rc, which was the strongest inhibitor of UGT1A9 activity. Potent inhibition against human UGT1A9 by ginsenoside Rc was demonstrated in this study (Tables 3 and 4). Ginsenoside Rc exhibited selective

\section{A Mycophenolic acid}
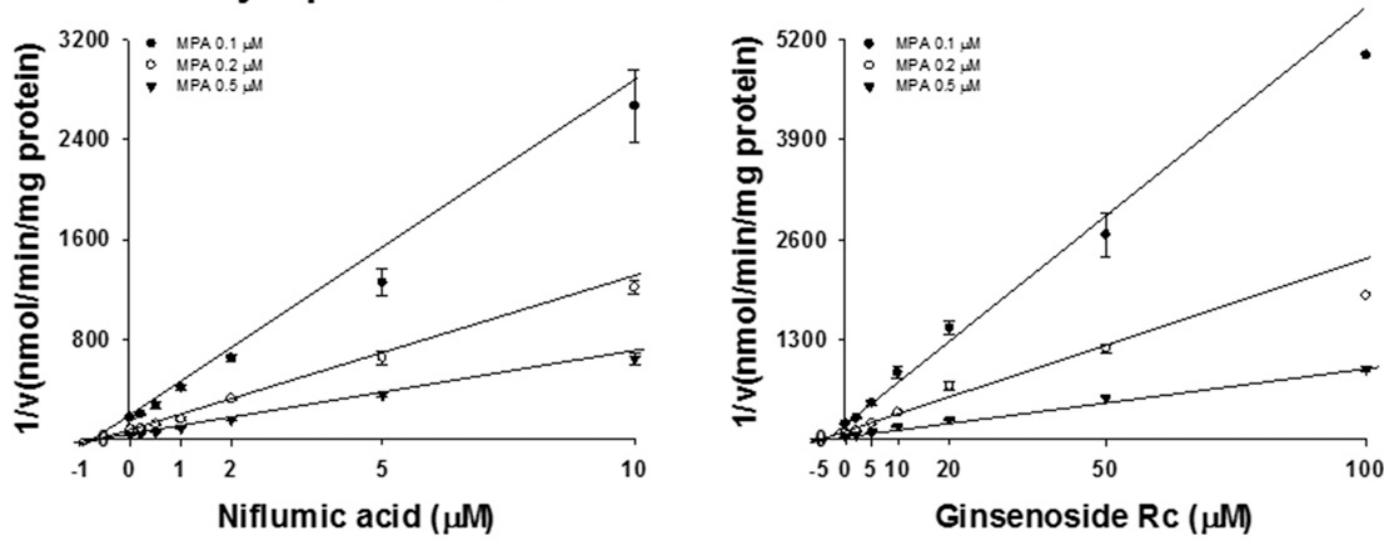

\section{B Propofol}
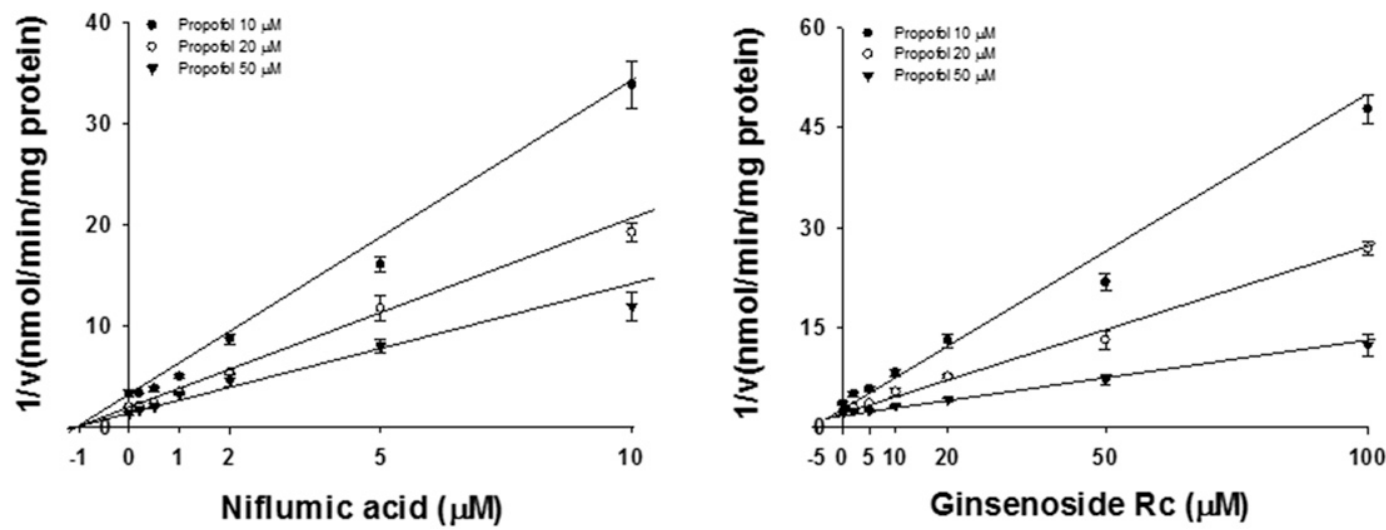

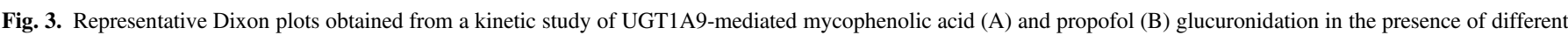

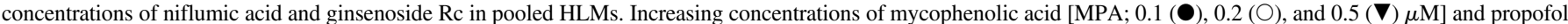

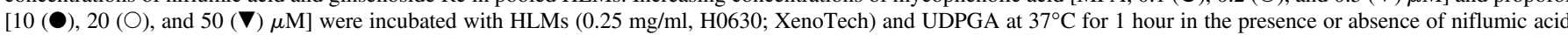

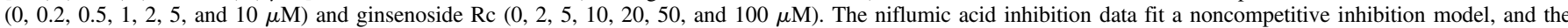
ginsenoside Rc inhibition data fit competitive and noncompetitive inhibition models. The data are means of triplicate measurements $(n=3)$. 


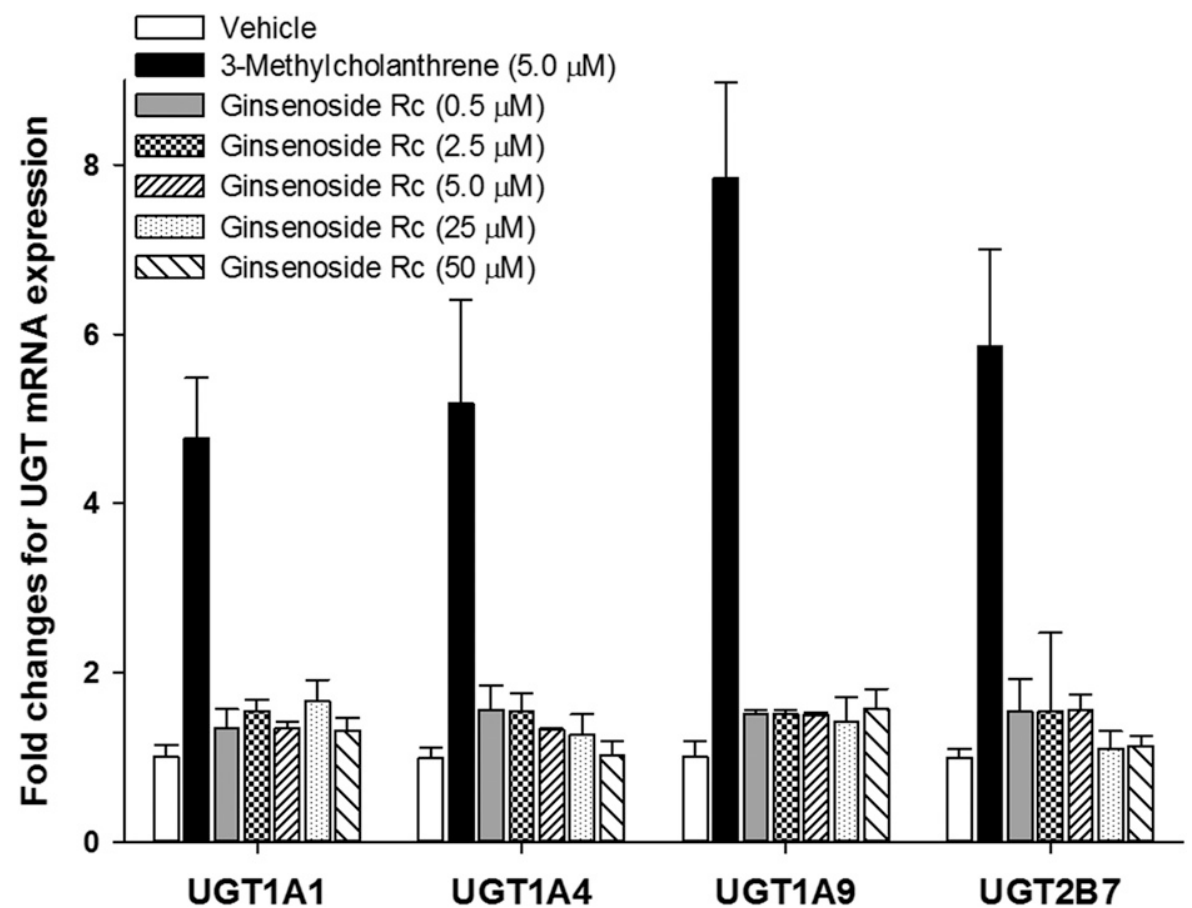

Fig. 4. Effect of ginsenoside Rc $(0.5,2.5,5.0,25$, and $50 \mu \mathrm{M})$ and 3-methylcholanthrene $(5.0 \mu \mathrm{M})$ on the mRNA levels of UGT1A1, 1A4, 1A9, and 2B7 after 48-hour treatment in the cryopreserved human hepatocytes (HFC520). The fold changes for UGT mRNA levels of ginsenoside Rc treatment were calculated by comparing with vehicle treatment (DMSO 0.1\%), and glyceraldehyde-3-phosphate dehydrogenase was used as an endogenous internal standard. The data are shown as means of triplicate measurements $(n=3)$. inhibition against UGT1A9 activity in HLMs and rUGT1A9 activity, with $\mathrm{IC}_{50}$ values of 6.34 and $6.38 \mu \mathrm{M}$, respectively. UGT1A9, a phase II metabolizing enzyme located in the endoplasmic reticulum, contributed to the glucuronidation reactions of endogenous compounds and xenobiotics, including arbidol (Song et al., 2013), dapagliflozin (Pattanawongsa et al., 2015), ethanol (Al Saabi et al., 2013), mefenamic acid (Gaganis et al., 2007), propofol (Court, 2005), and sorafenib (Peer et al., 2012). Therefore, new chemical entities with a backbone scaffold similar to ginsenoside Rc should be evaluated for potential UGT1A9 inhibitory effects during drug development. Some clinical drug interactions due to UGT1A9 inhibition have been reported. For example, coadministration with mefenamic acid has been shown to increase total dapagliflozin exposure (area under the concentration-time curve from time 0 to infinity) by $51 \%$ in healthy subjects (Kasichayanula et al., 2013).

Further inhibition kinetics studies using HLMs suggested that ginsenoside Rc competitively and noncompetitively inhibited UGT1A9mediated propofol and mycophenolic acid glucuronidation activities, with $K_{\mathrm{i}}$ values of 2.83 and $3.31 \mu \mathrm{M}$, respectively (Fig. 3), which are comparable to the $K_{\mathrm{i}}$ values of canagliflozin $(3.0 \mu \mathrm{M})$ (Pattanawongsa et al., 2015) and hesperetin (3.41 $\mu \mathrm{M})$ (Liu et al., 2016). The inhibitory potency of ginsenoside Rc was weaker than that of niflumic acid, with $K_{\mathrm{i}}$ values ranging $0.70-1.07 \mu \mathrm{M}$ (Fig. 3; Table 5). It was estimated that an in vivo interaction potential via the inhibition of UGT would likely

TABLE 6

Ratios of ginsenoside Rc in hepatocytes to culture medium in the cryopreserved human hepatocytes (HFC520) after 48-hour treatment of ginsenoside Rc

\begin{tabular}{lccc}
\hline & \multicolumn{3}{c}{ Ginsenoside Rc } \\
\cline { 2 - 4 } Hepatocytes-to-Medium Ratio & $0.5 \mu \mathrm{M}$ & $5.0 \mu \mathrm{M}$ & $25 \mu \mathrm{M}$ \\
\hline$\# 1$ & 1.54 & 0.83 & 1.14 \\
$\# 2$ & 1.61 & 0.95 & 1.82 \\
$\# 3$ & 1.38 & 1.11 & 1.25 \\
Average & 1.51 & 0.96 & 1.41 \\
S.D. & 0.12 & 0.14 & 0.37 \\
\hline
\end{tabular}

occur if the ratio of inhibitor $C_{\max } / K_{\mathrm{i}}$ were greater than 1 and would be possible if it were between 1.0 and 0.1 (Bjornsson et al., 2003; Bachmann and Lewis, 2005). Based on ginsenoside Rc's maximum concentrations $(0.09 \mu \mathrm{M})$ in human blood after repeated administration of red ginseng extract ( $>60 \%$ dried ginseng, once daily for 2 weeks; content: $23.0 \mathrm{mg}$ of ginsenoside $\mathrm{Rb} 1,11.4 \mathrm{mg}$ of ginsenoside $\mathrm{Rb} 2,13.0 \mathrm{mg}$ of ginsenoside Rc, $6.6 \mathrm{mg}$ of ginsenoside Rd, $6.2 \mathrm{mg}$ of ginsenoside $\mathrm{Re}, 2.8 \mathrm{mg}$ of ginsenoside $\mathrm{Rg} 1,8.0 \mathrm{mg}$ of ginsenoside Rh1, and 14.1 mg of ginsenoside Rg3) (Choi et al., 2019), the values of $C_{\max } / K_{\mathrm{i}}$ after a repeated administration of red ginseng extract in human were more than 0.032 from the data of HLMs $\left(K_{\mathrm{i}}=2.83 \mu \mathrm{M}\right)$, indicating that ginsenoside $\mathrm{Rc}$ has no drug interaction potential in humans (Liu et al., 2016). The area under the plasma drug concentrationtime curve of the coadministered drug may not change based on the in vitro-in vivo extrapolation prediction equation of Fang et al. (2013) when red ginseng extract is coadministered with drugs that are mainly eliminated via UGT1A9 in humans. The ginsenoside Rc concentration in liver is important to predict in vivo drug interaction potential of ginsenoside Rc. We evaluated the accumulation potential of ginsenoside Rc in liver using human hepatocytes. The concentration of ginsenoside Rc in the hepatocytes was similar to that of medium (Table 6). In addition, we also evaluated the induction potential of ginsenoside Rc on four UGT isoforms using human hepatocytes. Ginsenoside Rc did not significantly increase the mRNA expression of four UGT isoforms (Fig. 4). Taken together, these results suggest that ginsenoside Rc would not cause clinically relevant herb-drug interactions via the induction or inhibition of UGT enzymes following the usual dose of red ginseng extract (equivalent to $13.0 \mathrm{mg}$ of ginsenoside Rc) in humans.

Ginsenoside $\mathrm{Rc}$ is an active component of ginseng. The main root of $P$. ginseng contains high amounts of ginsenoside $\mathrm{Rb} 1, \mathrm{Rc}$, and $\mathrm{Rg} 1$, whereas the leaf contains high amounts of ginsenoside Rb3 and Rh1 (Attele et al., 2002; Noh et al., 2009; Kang and Kim, 2016). In commercially available red ginseng products, ginsenoside Rc has been found to be the second most abundant ginsenoside (Lee et al., 2015). Ginsenoside Rc has been shown to have analgesic, antiallergic, 
antitumor, and sedative effects (Chu et al., 2013). Ginsenoside Rc has also been proposed as a strong antidiabetic agent because it remarkably enhances glucose uptake by inducing reactive oxygen species generation (Lee et al., 2010). In addition, ginseng extract is frequently administered as a health supplement along with cancer chemotherapy based on its ability to restore vitality and stimulate the immune system (Lee and Kim, 2014). A high dose of red ginseng extract and fermented red ginseng extract ( $3 \mathrm{~g} /$ day for more than 2 months) in combination with platinum-based chemotherapy also improved cancer-related fatigue, emotional symptoms, and quality of life scores and reduced anticancer drug-related toxicity (Jiang et al., 2017; Kim et al., 2017). Therefore, clinical herb-drug interaction between ginsenoside Rc and UGT1A9 following the administration of high-dose ginseng adjuvant therapy or ginsenoside Rc single formulation needs to be further evaluation. Enzyme-selective inhibitors represent the most valuable experimental tools for the identification of drug-metabolizing enzymes responsible for drug metabolism in vitro. To evaluate the selectivity of ginsenoside Rc, we examined the inhibitory activities of ginsenoside Rc and niflumic acid against nine UGT isoforms, including UGTs 1A1, 1A3, 1A4, 1A6, 1A9, 2B, 2B7, 2B15, and 2B17, in $0.25 \mathrm{mg} / \mathrm{ml}$ recombinant UGTs. Ginsenoside Rc inhibited UGT1A1, UGT1A3, UGT1A9, UGT2B4, UGT2B7, and UGT2B15; however, the greatest inhibition was observed with UGT1A9, with an $\mathrm{IC}_{50}$ value of $6.38 \mu \mathrm{M}$ (Table 4). Ginsenoside Rc showed a 12.9-fold selectivity for UGT1A9 inhibition similar to niflumic acid, a well known selective UGT1A9 inhibitor (Miners et al., 2011), which showed a 12.5-fold selectivity (Table 4). The selectivity of ginsenoside Rc for UGT1A9 inhibition was higher than that of previously reported UGT1A9 inhibitors, including canagliflozin (3-fold), dapagliflozin (5-fold) (Pattanawongsa et al., 2015), and magnolol (10-fold) (Zhu et al., 2012). At $50 \mu \mathrm{M}$ ginsenoside Rc concentration, approximately 15 -fold greater than the $K_{\mathrm{i}}$ value, ginsenoside Rc was found to inhibit UGT1A9 by $90.7 \%$ and only slightly affected the enzyme activities of the other UGT isoforms tested (Fig. 2B). Ginsenoside Rc weakly inhibited UGT1A1 and UGT2B15 activities $(<32.5 \%)$ at concentrations of $50 \mu \mathrm{M}$ in recombinant UGT isoforms (Fig. 2B). Additionally, at $50 \mu \mathrm{M}$, ginsenoside Rc inhibited none of the other UGT isoform-specific activities above $12.0 \%$, except for UGT1A9 $(>91.5 \%)$ in HLMs (Fig. 2A), indicating that ginsenoside Rc could be used as a selective UGT1A9 inhibitor in reaction phenotyping studies. Notably, at $50 \mu \mathrm{M}$, niflumic acid, a well known selective UGT1A9 inhibitor, inhibited UGT1A1 and UGT2B15 activities by $73.9 \%$ and $59.7 \%$, respectively, in recombinant UGT isoforms.

In conclusion, we report that ginsenoside Rc is a selective UGT1A9 inhibitor. Ginsenoside Rc, when evaluated for mycophenolic acid glucuronidation inhibitory activity against UGT1A9 as well as eight other UGTs, exhibited a 13-fold selectivity for UGT1A9. Along with hecogenin, niflumic acid, and fluconazole, which are selective inhibitors of UGT1A4, UGT1A9, and UGT2B7, respectively, ginsenoside Rc could be useful as a selective UGT1A9 inhibitor in UGT reaction phenotyping studies such as niflumic acid when HLMs are used as the enzyme source. Additionally, ginsenoside Rc would not cause clinically relevant pharmacokinetic herb-drug interactions with other coadministered drugs metabolized by UGT1A9 when administered with the usual dose of red ginseng extract in humans.

\section{Authorship Contributions}

Participated in research design: Liu.

Conducted experiments: H. Lee, Heo, G.-H. Lee, Park, Jang, Kim, Kwon, Song.

Contributed new reagents or analytic tools: Song, Liu.

Performed data analysis: H. Lee, Song, Liu.

Wrote or contributed to the writing of the manuscript: H. Lee, Song, Liu.

\section{References}

Al Saabi A, Allorge D, Sauvage FL, Tournel G, Gaulier JM, Marquet P, and Picard N (2013) Involvement of UDP-glucuronosyltransferases UGT1A9 and UGT2B7 in ethanol glucuronidation, and interactions with common drugs of abuse. Drug Metab Dispos 41:568-574.

Attele AS, Wu JA, and Yuan CS (1999) Ginseng pharmacology: multiple constituents and multiple actions. Biochem Pharmacol 58:1685-1693.

Attele AS, Zhou YP, Xie JT, Wu JA, Zhang L, Dey L, Pugh W, Rue PA, Polonsky KS, and Yuan CS (2002) Antidiabetic effects of Panax ginseng berry extract and the identification of an effective component. Diabetes 51:1851-1858.

Bachmann KA and Lewis JD (2005) Predicting inhibitory drug-drug interactions and evaluating drug interaction reports using inhibition constants. Ann Pharmacother 39:1064-1072.

Bilgi N, Bell K, Ananthakrishnan AN, and Atallah E (2010) Imatinib and Panax ginseng: a potential interaction resulting in liver toxicity. Ann Pharmacother 44:926-928.

Bjornsson TD, Callaghan JT, Einolf HJ, Fischer V, Gan L, Grimm S, Kao J, King SP, Miwa G, Ni L, et al.; Pharmaceutical Research and Manufacturers of America (PhRMA) Drug Metabolism/Clinical Pharmacology Technical Working Group; ; FDA Center for Drug Evaluation and Research (CDER) (2003) The conduct of in vitro and in vivo drugdrug interaction studies: a Pharmaceutical Research and Manufacturers of America (PhRMA) perspective. Drug Metab Dispos 31:815-832.

Chen CF, Chiou WF, and Zhang JT (2008) Comparison of the pharmacological effects of Panax ginseng and Panax quinquefolium. Acta Pharmacol Sin 29:1103-1108.

Cheng TO (2005) Ginseng and other herbal medicines that interact with warfarin. Int J Cardiol 104:227.

Choi EJ, Park JB, Yoon KD, and Bae SK (2014) Evaluation of the in vitro/in vivo potential of five berries (bilberry, blueberry, cranberry, elderberry, and raspberry ketones) commonly used as herbal supplements to inhibit uridine diphospho-glucuronosyltransferase. Food Chem Toxicol 72:13-19.

Choi MK, Jin S, Jeon JH, Kang WY, Seong SJ, Yoon YR, Han YH, and Song IS (2019) Tolerability and pharmacokinetics of ginsenosides Rb1, Rb2, Rc, Rd, and compound $\mathrm{K}$ after single or multiple administration of red ginseng extract in human beings. J Ginseng Res DOI: 10.1016/j.jgr.2018.1010.1006.

Chu Y, Zhang HC, Li SM, Wang JM, Wang XY, Li W, Zhang LL, Ma XH, Zhou SP, Zhu YH, et al. (2013) Determination of ginsenoside Rc in rat plasma by LC-MS/MS and its application to a pharmacokinetic study. J Chromatogr B Analyt Technol Biomed Life Sci 919-920:75-78.

Court MH (2005) Isoform-selective probe substrates for in vitro studies of human UDP-glucuronosyltransferases. Methods Enzymol 400:104-116.

Fang ZZ, Cao YF, Hu CM, Hong M, Sun XY, Ge GB, Liu Y, Zhang YY, Yang L, and Sun HZ (2013) Structure-inhibition relationship of ginsenosides towards UDP-glucuronosyltransferases (UGTs). Toxicol Appl Pharmacol 267:149-154.

Gaganis P, Miners JO, and Knights KM (2007) Glucuronidation of fenamates: kinetic studies using human kidney cortical microsomes and recombinant UDP-glucuronosyltransferase (UGT) 1A9 and 2B7. Biochem Pharmacol 73:1683-1691.

Hauser B, Deschner T, and Boesch C (2008) Development of a liquid chromatography-tandem mass spectrometry method for the determination of 23 endogenous steroids in small quantities of primate urine. J Chromatogr B Analyt Technol Biomed Life Sci 862:100-112.

Ito M, Yamamoto K, Sato H, Fujiyama Y, and Bamba T (2001) Inhibitory effect of troglitazone on glucuronidation catalyzed by human uridine diphosphate-glucuronosyltransferase 1A6. Eur J Clin Pharmacol 56:893-895.

Jiang SL, Liu HJ, Liu ZC, Liu N, Liu R, Kang YR, Ji JG, Zhang C, Hua BJ, and Kang SJ (2017) Adjuvant effects of fermented red ginseng extract on advanced non-small cell lung cancer patients treated with chemotherapy. Chin J Integr Med 23:331-337.

Jin S, Jeon JH, Lee S, Kang WY, Seong SJ, Yoon YR, Choi MK, and Song IS (2019) Detection of 13 ginsenosides (Rb1, Rb2, Rc, Rd, Re, Rf, Rg1, Rg3, Rh2, F1, compound K, $20(S)$-Protopanaxadiol, and 20(S)-Protopanaxatriol) in human plasma and application of the analytical method to human pharmacokinetic studies following two week-repeated administration of red ginseng extract. Molecules $\mathbf{2 4}$.

Joo J, Lee B, Lee T, and Liu KH (2014) Screening of six UGT enzyme activities in human liver microsomes using liquid chromatography/triple quadrupole mass spectrometry. Rapid Commun Mass Spectrom 28:2405-2414.

Kang OJ and Kim JS (2016) Comparison of ginsenoside contents in different parts of Korean ginseng (Panax ginseng C.A. Meyer). Prev Nutr Food Sci 21:389-392.

Kasichayanula S, Liu X, Griffen SC, Lacreta FP, and Boulton DW (2013) Effects of rifampin and mefenamic acid on the pharmacokinetics and pharmacodynamics of dapagliflozin. Diabetes Obes Metab 15:280-283.

Kawase A, Takeshita F, Yamada A, Murata K, Matsuda H, Samukawa K, and Iwaki M (2009) Ginseng extracts facilitate cytochrome $\mathrm{P} 450$ xenobiotic metabolism in primary cultures of rat hepatocytes. J Health Sci 55:809-813.

Kiang TK, Ensom MH, and Chang TK (2005) UDP-glucuronosyltransferases and clinical drugdrug interactions. Pharmacol Ther 106:97-132.

Kim D, Zheng YF, Min JS, Park JB, Bae SH, Yoon KD, Chin YW, Oh E, and Bae SK (2016) In vitro stereoselective inhibition of ginsenosides toward UDP-glucuronosyltransferase (UGT) isoforms. Toxicol Lett 259:1-10.

Kim HS, Kim MK, Lee M, Kwon BS, Suh DH, and Song YS (2017) Effect of red ginseng on genotoxicity and health-related quality of life after adjuvant chemotherapy in patients with epithelial ovarian cancer: a randomized, double blind, placebo-controlled trial. Nutrients 9.

Kim SJ, Choi S, Kim M, Park C, Kim GL, Lee SO, Kang W, and Rhee DK (2018) Effect of Korean Red Ginseng extracts on drug-drug interactions. J Ginseng Res 42:370-378.

Lee $\mathrm{CH}$ and Kim JH (2014) A review on the medicinal potentials of ginseng and ginsenosides on cardiovascular diseases. J Ginseng Res 38:161-166.

Lee MS, Hwang JT, Kim SH, Yoon S, Kim MS, Yang HJ, and Kwon DY (2010) Ginsenoside Rc, an active component of Panax ginseng, stimulates glucose uptake in $\mathrm{C} 2 \mathrm{C} 12$ myotubes through an AMPK-dependent mechanism. J Ethnopharmacol 127:771-776.

Lee SM, Bae BS, Park HW, Ahn NG, Cho BG, Cho YL, and Kwak YS (2015) Characterization of Korean Red Ginseng (Panax ginseng Meyer): history, preparation method, and chemical composition. J Ginseng Res 39:384-391.

Liu D, Wu J, Xie H, Liu M, Takau I, Zhang H, Xiong Y, and Xia C (2016) Inhibitory effect of hesperetin and naringenin on human UDP-glucuronosyltransferase enzymes: implications for herb-drug interactions. Biol Pharm Bull 39:2052-2059. 
Malati CY, Robertson SM, Hunt JD, Chairez C, Alfaro RM, Kovacs JA, and Penzak SR (2012) Influence of Panax ginseng on cytochrome P450 (CYP)3A and P-glycoprotein (P-gp) activity in healthy participants. J Clin Pharmacol 52:932-939.

Mendonza A, Hanna I, Meyers D, Koo P, Neelakantham S, Zhu B, Majumdar T, Rebello S, Sunkara G, and Chen J (2016) Assessment of pharmacokinetic drug-drug interaction between pradigastat and atazanavir or probenecid. J Clin Pharmacol 56:355-364.

Miners JO, Bowalgaha K, Elliot DJ, Baranczewski P, and Knights KM (2011) Characterization of niflumic acid as a selective inhibitor of human liver microsomal UDP-glucuronosyltransferase 1A9: application to the reaction phenotyping of acetaminophen glucuronidation. Drug Metab Dispos 39:644-652.

Mohamed ME and Frye RF (2011) Inhibitory effects of commonly used herbal extracts on UDP-glucuronosyltransferase 1A4, 1A6, and 1A9 enzyme activities. Drug Metab Dispos 39: $1522-1528$.

Mroz A, Ryska I, Sominko H, Bejrowska A, and Mazerska Z (2018) Drug-drug interaction potential of antitumor acridine agent C-1748: the substrate of UDP-glucuronosyltransferases 2B7, 2B17 and the inhibitor of 1A9 and 2B7. Pharmacol Rep 70: 972-980.

Noh KH, Son JW, Kim HJ, and Oh DK (2009) Ginsenoside compound K production from ginseng root extract by a thermostable beta-glycosidase from Sulfolobus solfataricus. Biosci Biotechnol Biochem 73:316-321.

Oda S, Fujiwara R, Kutsuno Y, Fukami T, Itoh T, Yokoi T, and Nakajima M (2015) Targeted screen for human UDP-glucuronosyltransferases inhibitors and the evaluation of potential drugdrug interactions with zafirlukast. Drug Metab Dispos 43:812-818.

Ohno A, Saito Y, Hanioka N, Jinno H, Saeki M, Ando M, Ozawa S, and Sawada J (2004) Involvement of human hepatic UGT1A1, UGT2B4, and UGT2B7 in the glucuronidation of carvedilol. Drug Metab Dispos 32:235-239.

Pattanawongsa A, Chau N, Rowland A, and Miners JO (2015) Inhibition of human UDP-glucuronosyltransferase enzymes by canagliflozin and dapagliflozin: implications for drug-drug interactions. Drug Metab Dispos 43:1468-1476.

Peer CJ, Sissung TM, Kim A, Jain L, Woo S, Gardner ER, Kirkland CT, Troutman SM, English BC, Richardson ED, et al. (2012) Sorafenib is an inhibitor of UGT1A1 but is metabolized by UGT1A9: implications of genetic variants on pharmacokinetics and hyperbilirubinemia. Clin Cancer Res 18:2099-2107.

Phuc NM, Wu Z, O Y, Lee JH, Oh S, Song GY, and Liu KH (2017) LKY-047: first selective inhibitor of cytochrome P450 2J2. Drug Metab Dispos 45:765-769.

Qi LW, Wang CZ, and Yuan CS (2011) Ginsenosides from American ginseng: chemical and pharmacological diversity. Phytochemistry 72:689-699.

Sahai J, Gallicano K, Pakuts A, and Cameron DW (1994) Effect of fluconazole on zidovudine pharmacokinetics in patients infected with human immunodeficiency virus. J Infect Dis 169: 1103-1107.

Seo KA, Kim HJ, Jeong ES, Abdalla N, Choi CS, Kim DH, and Shin JG (2014) In vitro assay of six UDP-glucuronosyltransferase isoforms in human liver microsomes, using cocktails of probe substrates and liquid chromatography-tandem mass spectrometry. Drug Metab Dispos 42:1803-1810.

Seong SJ, Kang WY, Heo JK, Jo J, Choi WG, Liu KH, Lee S, Choi MK, Han YH, Lee HS, et al. (2018) A comprehensive in vivo and in vitro assessment of the drug interaction potential of red ginseng. Clin Ther 40:1322-1337.
Smith M, Lin KM, and Zheng YP (2001) An open trial of nifedipine-herb interactions: nifedipine with St. John's wort, ginseng, or Ginkgo biloba. Clin Pharmacol Ther 69:86-86.

Soars MG, Petullo DM, Eckstein JA, Kasper SC, and Wrighton SA (2004) An assessment of udp-glucuronosyltransferase induction using primary human hepatocytes. Drug Metab Dispos 32:140-148.

Song JH, Fang ZZ, Zhu LL, Cao YF, Hu CM, Ge GB, and Zhao DW (2013) Glucuronidation of the broad-spectrum antiviral drug arbidol by UGT isoforms. J Pharm Pharmacol 65:521-527.

Sten T, Finel M, Ask B, Rane A, and Ekström L (2009a) Non-steroidal anti-inflammatory drugs interact with testosterone glucuronidation. Steroids 74:971-977.

Sten T, Kurkela M, Kuuranne T, Leinonen A, and Finel M (2009b) UDP-glucuronosyltransferases in conjugation of 5alpha- and 5beta-androstane steroids. Drug Metab Dispos 37:2221-2227.

Tyler VE (2000) Herbal medicine: from the past to the future. Public Health Nutr 3 (4A):447-452

Uchaipichat V, Mackenzie PI, Elliot DJ, and Miners JO (2006) Selectivity of substrate (trifluoperazine) and inhibitor (amitriptyline, androsterone, canrenoic acid, hecogenin, phenylbutazone, quinidine, quinine, and sulfinpyrazone) "probes" for human udp-glucuronosyltransferases. Drug Metab Dispos 34:449-456.

Uchaipichat V, Mackenzie PI, Guo XH, Gardner-Stephen D, Galetin A, Houston JB, and Miners JO (2004) Human udp-glucuronosyltransferases: isoform selectivity and kinetics of 4-methylumbelliferone and 1-naphthol glucuronidation, effects of organic solvents, and inhibition by diclofenac and probenecid. Drug Metab Dispos 32:413-423.

Vaes LP and Chyka PA (2000) Interactions of warfarin with garlic, ginger, ginkgo, or ginseng: nature of the evidence. Ann Pharmacother 34:1478-1482.

You LY, Yu CN, Xie SG, Chen SQ, and Zeng S (2007) Stereoselective glucuronidation of carvedilol by Chinese liver microsomes. J Zhejiang Univ Sci B 8:756-764.

Zhang D, Chando TJ, Everett DW, Patten CJ, Dehal SS, and Humphreys WG (2005) In vitro inhibition of UDP glucuronosyltransferases by atazanavir and other HIV protease inhibitors and the relationship of this property to in vivo bilirubin glucuronidation. Drug Metab Dispos 33: 1729-1739.

Zheng YF, Bae SH, Choi EJ, Park JB, Kim SO, Jang MJ, Park GH, Shin WG, Oh E, and Bae SK (2014) Evaluation of the in vitro/in vivo drug interaction potential of BST204, a purified dry extract of ginseng, and its four bioactive ginsenosides through cytochrome P450 inhibition/ induction and UDP-glucuronosyltransferase inhibition. Food Chem Toxicol 68:117-127.

Zhu L, Ge G, Liu Y, He G, Liang S, Fang Z, Dong P, Cao Y, and Yang L (2012) Potent and selective inhibition of magnolol on catalytic activities of UGT1A7 and 1A9. Xenobiotica 42 1001-1008.

Address correspondence to: Kwang-Hyeon Liu, BK21 Plus KNU Multi-Omics based Creative Drug Research Team, College of Pharmacy and Research Institute of Pharmaceutical Sciences, Kyungpook National University, Daegu 41566, Korea. E-mail: dstlkh@knu.ac.kr; or Im-Sook Song, College of Pharmacy and Research Institute of Pharmaceutical Sciences, Kyungpook National University, Daegu 41566, Korea. E-mail: isssong@knu.ac.kr 